\title{
Protection Motivation Theory and Social Distancing Behaviour in Response to a Simulated Infectious Disease Epidemic
}

Epidemics of respiratory infectious disease remain one of the most serious health risks facing the population. Non-pharmaceutical interventions (e.g., hand-washing or wearing face masks) can have a significant impact on the course of an infectious disease epidemic. The current study investigated whether protection motivation theory (PMT) is a useful framework for understanding social distancing behaviour (i.e. the tendency to reduce social contacts) in response to a simulated infectious disease epidemic. There were 230 participants (109 males, 121 females, mean age 32.4 years) from the general population who completed self-report measures assessing the components of PMT (Milne, Orbell \& Sheeran, 2002). In addition, participants completed a computer game which simulated an infectious disease epidemic in order to provide a measure of social distancing behaviour (Maharaj, McCaldin \& Kleczkowski, 2011). The regression analyses revealed that none of the PMT variables were significant predictors of social distancing behaviour during the simulation task. However, fear $(\beta=.218, p<.001)$, response-

efficacy $(\beta=.175, p<.01)$ and self-efficacy $(\beta=0.251, p<0001)$ were all significant predictors of intention to engage in social distancing behaviour. Overall, the PMT variables (and demographic factors) explain $21.2 \%$ of the variance in intention. The findings demonstrated that PMT was a useful framework for understanding intention to engage in social distancing behaviour, but not actual behaviour during the simulated epidemic. These findings may reflect an intentionbehaviour gap in relation to social distancing behaviour.

Keywords: infectious disease; protection motivation; social distancing 


\section{Introduction}

Adopting protective behaviours (e.g. hand hygiene, social distancing, mask wearing) in response to respiratory infectious diseases can have a significant impact on the course of an epidemic (Teasdale et al., 2014). Health behaviour models, such as, Protection Motivation Theory (PMT; Rogers, 1975; 1983), or Theory of Planned Behaviour (Ajzen, 1991), provide useful frameworks for understanding behavioural choices during such epidemics. For example, the PMT components of perceived severity, perceived vulnerability, and response-efficacy were linked to undertaking behaviours to protect against infectious diseases (Bish \& Michie, 2010). In addition, research on the 2009 pandemic demonstrated the importance of perceived severity and response-efficacy in predicting vaccination uptake (e.g., Ashbaugh et al., 2013). Recently, Timpka at al. (2014) found that coping appraisal was associated with intention to perform health protective behaviours during an influenza outbreak.

Social distancing, when individuals reduce the number of social contacts they make in response to the presence of disease, is an important health-protective behaviour. Teasdale et al. (2012) used PMT to examine intentions to perform protective behaviours (including social distancing behaviour) during a hypothetical epidemic. They found that intending to stay at home during the epidemic (i.e. social distancing behaviour) was associated with all of the PMT components, with self-efficacy emerging as the single strongest predictor of intending to engage in protective behaviour during a hypothetical epidemic.

The use of hypothetical epidemics is common as investigating the predictors of behaviour change during infectious diseases in the real world can be difficult. It is not feasible to carry out experiments by starting epidemics and opportunistic data collection during naturally occurring epidemics can be difficult to carry out in practice. One novel way of investigating the problem is via scenario-based computer game tasks. These tasks may represent a useful way of measuring 
how people respond to hypothetical infectious disease outbreaks (Balicer, 2007; Chen et al., 2013). In the present study, our methodology involves participants playing a computer game in which they play the role of an individual in a neighbourhood that is experiencing an epidemic, and must make decisions about how many people to contact (Delaney et al., 2013). The current study investigates the utility of PMT in explaining intention to engage in social distancing behaviour, and actual social distancing behaviour employed during a simulated infectious disease.

\section{Method}

Participants and Procedure

In a cross-sectional design, 230 participants were recruited from Glasgow Science Centre and a University in Scotland (109 males, 121 females, mean age: 32.4 years). Ethical approval was obtained from the institutional review board. Each participant provided written informed consent, gave demographic information, and completed the following self-report measures and computer game task.

\section{Measures}

Protection Motivation Theory

Measures of PMT variables (perceived severity, perceived vulnerability, fear, response efficacy, self-efficacy, response cost, intention) were assessed with a 15-item questionnaire (based on Milne, Orbell, and Sheeran, 2002), which was modified in order to relate to an infectious disease epidemic (e.g., 'If I were to develop an infectious disease I would suffer a lot of unpleasant symptoms'). All of the questions were rated on a scale ranging from 1 (strongly disagree) to 5 (strongly agree). 


\section{Scenario-Based Computer Game}

Social distancing behaviour was measured through participation in a computer game (Maharaj, McCaldin and Kleczkowski, 2011; Delaney et al., 2013). The two-dimensional game represents an epidemic scenario, linked to an agent-based simulation of an epidemic spreading through a large population. The participant plays the role of a susceptible individual in a society experiencing an epidemic. The instructions inform participants that there is 'an epidemic of a highly infectious disease' (a specific epidemic is not identified and participants are not given any information about specific disease characteristics). The infection pressure in the participant's neighbourhood is indicated by the relative numbers of green (uninfected) and red (infected) figures shown on screen. The participant then clicks on a blue circle which lights up signalling that the participant can drag it down to indicate how many people they want to meet that day. The game interface records the current infection pressure and the value of the participant's choice. These are used to calculate a single value representing his/her social distancing behaviour (Maharaj \& Kleczkowski, 2012). The agent-based simulation contains 2500 agents, one of which is controlled by the player and the rest are computer-controlled. Initially, 150 randomly chosen individuals (6\%) are infected, and the others are susceptible. Thereafter, the computer-controlled agents mirror the social distancing behaviour adopted by the participant (Kleczkowski et al., 2015). The game comes to an end when the participant becomes infected or when the epidemic ends, which happens when all infected individuals in the simulated population recover, or when a maximum number of periods is reached.

\section{Statistical Analyses}

Correlations were used to examine the associations between the PMT variables and social distancing behaviour. In addition, hierarchical multiple regression analysis was used to examine those factors that were predictive of intention to engage in social distancing behaviour during an infectious disease epidemic. 


\section{Results}

Descriptive statistics and correlation analysis

Descriptive statistics for the PMT variables demonstrate that response levels on all variables were moderate. Simulation results showed that the social distancing response was weak, tending to prolong the epidemic instead of stopping it (Kleczkowski et al., 2015). Analysis showed that social distancing behaviour was not significantly correlated with any of the PMT variables. However, intention to engage in social distancing behaviour was significantly correlated with fear $(r=.258, p<.01)$, response-efficacy $(r=.223, p<.01)$, self-efficacy $(r=.340$, $p<.01)$, and age $(r=-.184, p<.01)$. Intention to engage in social distancing was not correlated with social distancing behaviour during the computer game task $(r=.121, p=.067)$ (see Table 1).

\section{Insert Table 1}

\section{Hierarchical multiple regression}

As shown in Table 2, a hierarchical regression analysis of intention to engage in social distancing behaviour was conducted. The demographic (age and gender) variables were entered in the first step and accounted for a significant amount (3.6\%) of the variance in social distancing behaviour $\left(R^{2}=.036\right)$. Next, those PMT variables that were significantly correlated with intention (i.e. fear, response-efficacy, and self-efficacy) were entered at Step 2, and significantly increased the amount of variance explained by $17.8 \%$. The final model explained $21.4 \%$ of the variance in intention $\left(R^{2}=.214\right)$, with age $(\beta=-.170, p<.01)$, fear $(\beta=.218$, $p<.001)$, response-efficacy $(\beta=.175, p<.01)$ and self-efficacy $(\beta=0.251, p<.001)$ emerging as significant predictors. 
Insert Table 2

\section{Discussion}

The findings demonstrated no association between PMT variables and social distancing behaviour during the computer game scenario. However, three PMT factors (fear, responseefficacy, and self-efficacy) were significant predictors of intention to engage in social distancing. Previous research has also identified the utility of these variables in predicting behavioural responses to respiratory infectious diseases (e.g., Teasdale et al., 2012; Timpka et al., 2014). The current study found no association between social distancing behaviour and intention, representing an intention-behaviour gap. The evidence suggests that social distancing may be a behaviour that individuals find difficult to perform (e.g., Teasdale et al., 2014). Therefore, increasing perceptions of efficacy surrounding this behaviour will be key in order to employ it as an effective behavioural intervention.

There are several limitations of the study. We collected the data during the summer, when infectious illnesses are less prevalent than during winter, which may have influenced participants' ability to identify with the scenario in the game. In addition, the social distancing behaviour that we measured is hypothetical and may not accurately reflect real life behaviour. The use of hypothetical scenarios is common within the literature. However, these scenarios may be problematic as participants may find it difficult to project themselves into these situations. Further limitations relate to the use of the computer game task. Recent research points to the usefulness of games as a potential methodological tool for the collection of responses to infectious diseases (Balicer, 2007; Chen et al., 2013), and there has also been a recent increase in the use of games within the context of health-behaviour change (Primack et al., 2011). However, there are drawbacks of using such methods. Hypothetical game-based scenarios may not reflect real life situations; instead participants may be simply playing the 
game as a game. Another limitation relates to whether the current game is immersive enough. A higher level of immersion via three-dimensional simulated worlds may encourage participants to behave in a more true-to-life manner. There are also aspects of the game, such as the colour indication of the number of susceptible/infected individuals that provides information to participants that does not exist in the real world.

The present study has investigated social distancing behaviour using a novel computer game scenario that simulated an infectious disease outbreak. Three components of PMT (fear, response-efficacy and self-efficacy) were significantly associated with intention to engage in social distancing behaviour, but not to social distancing behaviour during the game.

Understanding the psychological factors involved in behavioural responses to infectious diseases is important for shaping interventions that may be used during infectious disease epidemics. The use of computer game tasks may represent a useful way of investigating these behavioural responses. 


\section{References}

Ajzen, I. (1991). The theory of planned behavior. Organisational Behaviour and Human Decision Processes, 50, 179-211.

Ashbaugh, A.R., Herbert, C.F., Saimon, E., Azoulay, N., Olivera-Figueroa, L. \& Brunet, A. (2013). The decision to vaccinate or not during the H1N1 pandemic: selecting the lesser of two evils? PLoS One, 8 (3).

Balicer, R.D. (2007). Modeling infectious diseases dissemination through online role playing games. Epidemiology, 18, 260-261.

Bish, A. \& Michie, S. (2010). Demographic and attitudinal determinants of protective behaviours during a pandemic: A review. British Journal of Health Psychology, 15, 797-824.

Chen, F., Griffith, A., Cottrell, A. \& Wong, Y-L. (2013). Behavioural responses to epidemics in an online experiment: Using virtual diseases to study human behavior. PLoS ONE, 8(1).

Delaney, L., Kleczkowski, A., Maharaj, S., Rasmussen, S. \& Williams, L. (2013). Reflections on a Virtual Experiment Addressing Human Behaviour During Epidemics. In Proceedings of the 2013 Summer Computer Simulation Conference (SCSC '13). Society for Modeling \& Simulation International, Vista, CA, Article 33.

Kleczkowski, A., Maharaj, S., Rasmussen, S., Williams, L. \& Cairns, N. (2015, under review). Spontaneous social distancing during epidemics: Evidence from a virtual experiment. 
Maharaj, S. \& Kleczkowski, A. (2012). Controlling epidemic spread by social distancing: do it well or not at all. BMC Public Health, 12:679.

Maharaj, S., McCaldin, T. \& Kleczkowski, A. (2011). A Participatory Simulation Model for Studying Attitudes to Infection Risk, In Proceedings of the 2011 Summer Computer Simulation Conference (SCSC '11). Society for Modeling \& Simulation International, Vista, CA, 8-13.

Milne, S., Orbell, S., \& Sheeran, P. (2002). Combining motivational and volitional interventions to promote exercise participation: Protection motivation theory and implementation intentions. British Journal of Health Psychology, 7, 163-184.

Primack, B.A., Carroll, M.V., McNamara, M., Klem, M.L., King, B., Rich, M., Chan, C.W. \& Nayak. (2012). Role of video games in improving health-related outcomes: A systematic review. American Journal of Preventive Medicine, 42, 630-638.

Rogers, R. W. (1975). A protection motivation theory of fear appeals and attitude change. Journal of Psychology, 91, 93-114.

Rogers, R.W. (1983). Cognitive and physiological processes in fear appeals and attitude change: A revised theory of protection motivation. In J. Cacioppo \& R. Petty (Eds.), Social Psychophysiology. New York: Guilford Press.

Teasdale, E., Yardley, L., Schlotz, W. \& Michie, S. (2012). The importance of coping appraisal in behavioural responses to pandemic flu. British Journal of Health Psychology, 17, 44-59. 
Teasdale, E., Santer, M., Geraghty, A.W., Little, P. \& Yardley, L. (2014). Public perceptions of non-pharmaceutical interventions for reducing transmission of respiratory infection: systematic review and synthesis of qualitative studies. BMC Public Health, 14:589.

Timpka, T., Spreco, A., Gurko, E., Eriksson, O., Dahlstrom, O., Stromgen, M., Ekberg, J., Pilemalm, S., Karlsson, D., Hinkula, J. \& Holm, E. (2014). Intentions to perform nonpharmaceutical protective behaviors during influenza outbreaks in Sweden: a cross-sectional study following mass vaccination campaign. PLoS One, 9 (3). 
Table 1: Correlations between PMT variables and social distancing behaviour employed during the computer game task

\begin{tabular}{|c|c|c|c|c|c|c|c|c|}
\hline Variable & 1 & 2 & 3 & 4 & 5 & 6 & 7 & 8 \\
\hline 1. Age & - & & & & & & & \\
\hline 2. Perceived & .079 & _ & & & & & & \\
\hline \multicolumn{9}{|l|}{ Severity } \\
\hline 3. Perceived & .068 & $.135^{\star}$ & - & & & & & \\
\hline \multicolumn{9}{|l|}{ Vulnerability } \\
\hline 4. Fear & -.040 & -.013 & .126 & - & & & & \\
\hline 5. Response & .021 & $.224^{* *}$ & .107 & -.050 & - & & & \\
\hline efficacy & & & & & & & & \\
\hline 6. Self- & -.042 & $.141^{*}$ & $.312^{* *}$ & $.197^{*}$ & $.228^{* *}$ & - & & \\
\hline \multicolumn{9}{|l|}{ efficacy } \\
\hline 7. Intention & $-.184^{* *}$ & .038 & .119 & $.258^{* *}$ & $.223^{* *}$ & $.340^{* *}$ & - & \\
\hline 8. Social & .059 & -.018 & -.016 & .008 & .049 & .081 & .121 & - \\
\hline \multicolumn{9}{|l|}{ Distancing } \\
\hline Mean & 32.4 & 5 & 5.99 & 15.48 & 2.27 & 14.33 & 3.07 & .28 \\
\hline SD & 14.22 & 1.48 & .94 & 5.23 & .948 & 2.09 & 1.1 & .24 \\
\hline
\end{tabular}

Note. ${ }^{*} p<.05,{ }^{* *} p<.01$ 
Table 2. Hierarchical regression analysis predicting social distancing intention by demographic and PMT variables

\begin{tabular}{llllll}
\hline & Variable & Total $\mathrm{R}^{2}$ & $\Delta \mathrm{R}^{2}$ for step & Final beta & $\mathrm{t}$ \\
\hline Step 1 & Age & .036 & $.036^{*}$ & -.170 & $-2.85^{* *}$ \\
& Gender & & & .056 & .935 \\
Step 2 & Fear & .214 & $.178^{* * *}$ & .218 & $3.55^{* * *}$ \\
& Response Efficacy & & & .175 & $2.83^{* *}$ \\
& Self-efficacy & & & .251 & $4.00^{* * *}$ \\
& & & &
\end{tabular}

Note. ${ }^{*} p<.05,{ }^{* *} p<.01,{ }^{* *} p<.001$ 